\title{
Synchronous Primary Tumors of Kidney and Bilateral Ovaries-A Diagnostic Challenge: Case Report
}

\author{
Singh K., Gupta D., Anjali T., Mudgal V K., Trivedi N. and Agarwal S.
}

\begin{abstract}
Multiple malignancies in the same patient account for $2 \%$ to $17 \%$ of all cancers. However, multiple synchronous primary tumours in the same patient are extremely rare. Most synchronous primary tumors involve the genitourinary and gastrointestinal tract, followed by both breast and genitourinary tract, and breast and gastrointestinal tract. Among gynaecological malignancies, synchronous primary carcinomas of the endometrium and ovary are the commonest. Synchronous primary neoplasm involving kidney and ovaries are extremely rare, with only few case reports in the literature; however, there are no reported cases in the Indian literature. We hereby report a case of 60 year old woman who underwent radical nephrectomy for renal cancer and primary cytoreductive surgery for her ovarian cancer. In this report we present the first case in the Indian literature of synchronous primary cancers in right kidney and bilateral ovaries. Based on the combination of factors (diagnosis, treatment and demographics), it is expected that in the course of the coming years, the prevalence of patients with multiple primaries will increase. Careful attention should be paid to the differential diagnosis between double primary and metastatic tumours, based on the pathologic, radiological and clinical characteristics.
\end{abstract}

Index Terms - Synchronous; Metachronous; Multiple Primaries; Genitourinary.

\section{INTRODUCTION}

Multiple primaries are defined as more than one synchronous or metachronous cancer in the same individual. For epidemiological studies, tumours are considered multiple primary malignancies if arising in different sites and/or are of different histology or morphology group [1]. The fact that patients may have multiple primary tumours is not new, as already in 1921 a report found $4.7 \%$ of multiple primaries in 3000 cases they studied [2]. The recent literature reports the frequency of multiple primaries to be in the range of $2-17 \%$ [3]. Most synchronous primary tumors involve the genitourinary and gastrointestinal tract, followed by breast and genitourinary tract and breast and

Published on June 5, 2020.

Kriti Singh, DNB Student, Department of Pathology, Regency hospital, Kanpur, India. (email: singh.kunnu25@gmail.com)

Deepti Gupta, Consultant (Pathology) Department of Pathology, Regency Hospital, Kanpur, India. (email: guptadeeps@gmail.com)

Anjali Tewari, Consultant Pathologist and Head of Department of Lab sciences, Regency Hospital, Kanpur, India. (email: anjalitew@gmail.com)

Vinod K.Mudgal, Consultant Surgical Oncologist, Regency Hospital, Kanpur, India. (email: dr.vinodmudgal@gmail.com)

Nupur Trivedi, Consultant Pathologist, Regency Hospital, Kanpur, India. (email: nupur.dr@gmail.com)

Shefali Agarwal, Consultant Pathologist, Regency Hospital , Kanpur, India. (email: shefali2300@gmail.com) gastrointestinal tract 4]. Synchronous primary neoplasms involving the kidney and ovaries are extremely rare, with only few case reports in the literature [5 however, there are no reported cases in the Indian literature. We hereby report the first case in the Indian literature of synchronous primary cancers in right kidney and bilateral ovaries.

\section{CASE REPORT}

A 60 year old woman with history of indigestion for 2-3 months and breathlessness for 15 days came to our tertiary care hospital in March 2019. She underwent all the relevant investigations.CA-125 was markedly raised measuring $707.60 \mathrm{u} / \mathrm{ml}$. USG whole abdomen revealed a mitotically active heteroechoic lesion of $54 \times 46 \mathrm{~mm}$ in the upper and mid pole of right kidney (?Renal Cell Carcinoma) and mild right sided pleural effusion with passive collapse of basal segments of right lung lower lobe. PLEURAL and ASCITIC FLUID cytologies were negative for malignant cells. CECT THORAX and WHOLE ABDOMEN confirmed the malignant features of the renal mass - showed ill defined soft tissue lesion measuring $6.8 \times 5.8 \mathrm{~cm}$ at upper and mid pole of right kidney with necrotic changes. CECT also showed post menopausal atrophic uterus with thick walled cystic lesion of $4.2 \times 3.2 \mathrm{~cm}$ in left adnexa (?Left ovarian complex cyst), right sided mild to moderate pleural effusion and underlying collapse consolidation. F-18 FDG PET CT scan revealed heterogenous enhancing mass lesion of $6.6 \times 5.3 \times 6.1 \mathrm{~cm}$ with central necrosis and peripheral increased uptake (SUV max 6.0) involving the upper half of Right Kidney and complex solid cystic mass lesions with $F D G$ avid solid components in $\mathrm{B} / \mathrm{L}$ adnexal regions. Bilateral ovaries were not visualised separately suggesting primary carcinoma of bilateral ovaries. She was admitted in our oncosurgery department and was operated for suspected kidney and bilateral ovarian malignancies. The patient underwent right radical nephrectomy for her renal cancer and primary cytoreductive surgery for the ovarian cancers, consisting of total abdominal hysterectomy, bilateral salpingo-oopherectomy, pelvic and retroperitoneal lymphadenectomy, omenectomy and multiple peritoneal biopsies.

On histopathological examination, right kidney revealed clear cell carcinoma (Fig. 1) with pTNM stage - pT1bN0 and bilateral ovaries showed papillary adenocarcinoma (Fig. 2) with pTNM stage - pT1cN0. Vaginal flap, left broad ligament, peritoneal deposits, epigastric peritoneum, omentum, right paracolic gutter peritoneum and paraaortic lymph nodes, were all negative for tumour metastasis and showed sarcoid like granulomas. Immunohistochemistry panel for ovarian mass revealed papillary serous adenocarcinoma; was immunoreactive for CK7, WT1, ER and non immunoreactive for CK20, RCC, CD10. Right kidney mass was immunoreactive to PAX8 and therefore, 
making a diagnosis of clear cell carcinoma. Broad ligament, peritoneal deposits, epigastric peritoneum, omentum, right paracolic gutter peritoneum and paraaortic lymph nodes showed granulomatous inflammation and were negative for tumor metastasis. Hence, a final diagnosis of synchronous primary tumours of kidney and bilateral ovaries was made.

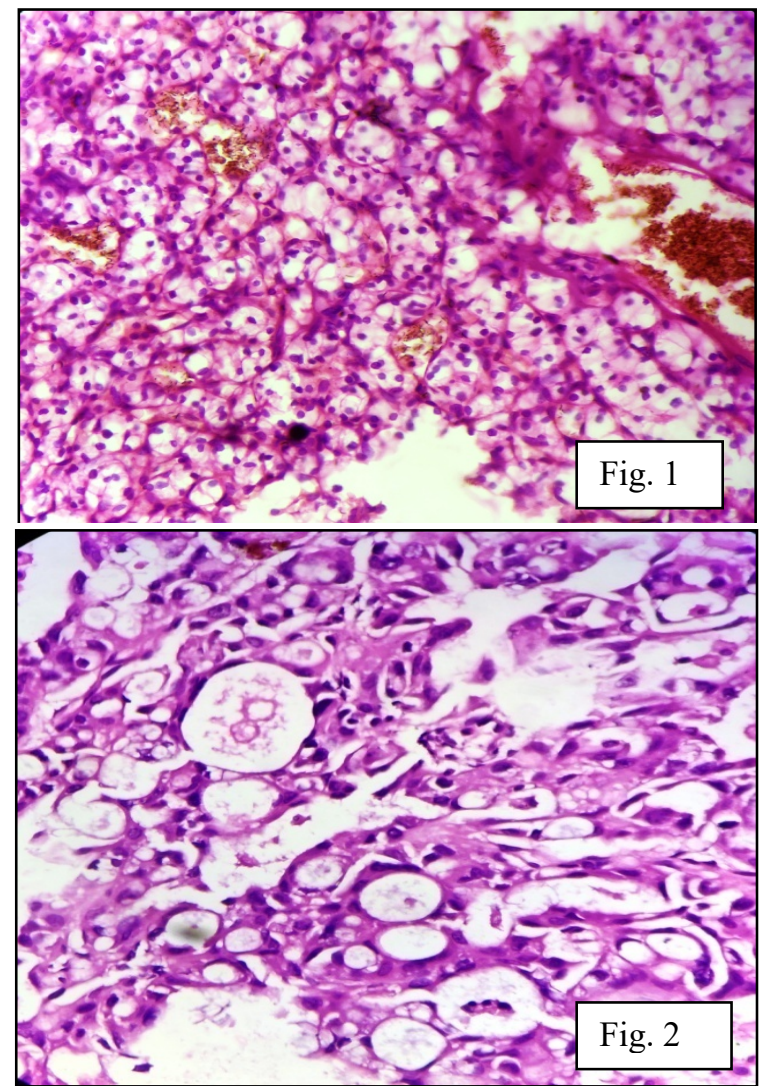

Fig. 1: H\&E stained section shows Clear Cell Carcinoma Kidney (x400)

Fig. 2: H\&E stained section shows Papillary Adenocarcinoma Ovary (x400)

\section{DISCUSSION}

Multiple primary cancer was described first by Warren and Gates in 1932 as (a) each tumour must be distinct (b) each tumour must exhibit marked features of malignancy (c) the probability of one lesion being a metastasis of the other must be excluded [6]. Synchronous and metachronous tumours are defined on the basis of length of interval between first and second cancer. Synchronous means tumours found simultaneously or within 6 months, while metachronous means more than 6 month interval [7]. Therefore, on the basis of this our current case classifies as synchronous double primary cancers. The reported prevalence of multiple primary malignancies varies between $2-17 \%$ [3].

The pathogenesis of synchronous malignancies in the same patient is poorly understood. However, common etiologies, such as inherited predisposition to cancer; cancer promoting aspects of lifestyle, hormonal and environmental factors; treatment of previous primary cancer; increased surveillance of cancer survivors; exposure to the same carcinogen or hormone, are often suspected [8].

Some RCCs have been thought to be hormone dependent. The association between RCC and steroid - hormone target organs (breast, ovaries and uterus) may be explained by such a hypothesis. In a study 17 cases of RCCs associated with second primary neoplasms occurring in steroid hormone target tissues were studied. Among them, 10 had associated breast carcinoma, 4 had endometrial carcinoma and only three had ovarian carcinoma [9].

With increasing use of more sophisticated imaging methods, namely PET-CT and whole body MRI, it is not uncommon to find suspicious lesions that might have not been detected by standard CT and/or bone scintigraphy. In a series of 1912 patients, additional suspicious malignant tumours were found in $4.1 \%$ of cases on PET-CT scans. $1.2 \%$ of these were confirmed by histology [10]. In a series of 200 patients with esophageal cancer, a total of $17 \%$ were found to have synchronous primary tumour on PET-CT, done for tumour staging [11]. The same happened in the present case where the ovarian malignancy was suspected on CECT and PET-CT.

In a case of suspected secondary primary, a histological confirmation should be pursued. With the advances in imaging and image guided biopsy techniques, an adequate biopsy is a must for tissue diagnosis. The clinicians must inform the pathologists about the primary cancer diagnosis and the primary tissue should also be available for comparison. This would help to differentiate between second primary and metastatic tumour, especially in the case of undifferentiated histologies.

Apart from diagnostic challenge, practical implications of the management of the patients with multiple primaries are important. The challenge is to find an anticancer therapy strategy that covers both cancer types without increased toxicity or relevant pharmacological interactions and without negative impact on the overall outcome.

Based on the combination of factors (diagnosis, treatment and demographics), it is expected that in the course of the coming years, the prevalence of patients with multiple primaries will increase. Careful attention should be paid to the differential diagnosis between double primary and metastatic tumours, based on the pathologic, radiological and clinical characteristics. Further research is needed, especially with regards to the areas of the treatment of patients with synchronous or metachronous multiple primary cancers.

\section{REFERENCES}

[1] Shah SA, Riaz U, Zahoor I, Jalil A, Zubair M. Carcinoma multiplex Journal of the College of Physicians and Surgeons--Pakistan: JCPSP. 2013 Apr;23(4):290-2.

[2] Owen LJ. Multiple malignant neoplasms. JAMA. 1921 May 14;76(20):1329-33.

[3] Coyte A, Morrison DS, McLoone P. Second primary cancer risk-the impact of applying different definitions of multiple primaries: results from a retrospective population-based cancer registry study. BMC Cancer. 2014 Dec 1;14(1):272.

[4] Irimie A, Achimas-Cadariu P, Burz C, Puscas E. Multiple primary malignancies--epidemiological analysis at a single tertiary institution. Journal of gastrointestinal and liver diseases: JGLD. 2010 Mar;19(1):69-73.

[5] Tsili AC, Charisiadi A, Koliopoulos G, Kamina S, Doukas M, Paraskevaidis E, Tsampoulas K. Synchronous primary tumors of the kidney and the ovaries: Imaging findings. Journal of Radiology Case Reports. 2008 Nov 27;2(5):2-8.

[6] Warren S, Gates O. Multiple primary malignant tumors. A survey of the literature and a statistical study. Am J cancer. 1932;16:1358-414.

[7] Moertel CG, Dockerty MB, Baggenstoss AH. Multiple primary malignant neoplasms. I. Introduction and presentation of data. Cancer. 1961 Mar;14(2):221-30. 
[8] Schottenfeld D, Berg J. Incidence of multiple primary cancers. IV. Cancers of the female breast and genital organs. Journal of the National Cancer Institute. 1971 Jan 1;46(1):161-70.

[9] Di Silverio F, Sciarra A, Flammia GP, Mariani M, De Vico A. Multiple primary tumors: 17 cases of renal-cell carcinoma associated with primary tumors involving different steroid-hormone target tissues. World journal of urology. 1997 Jun 1;15(3):203-9.

[10] Ishimori T, Patel PV, Wahl RL. Detection of unexpected additional primary malignancies with PET/CT. Journal of Nuclear Medicine. 2005 May 1;46(5):752-7.

[11] Miyazaki T, Sohda M, Higuchi T, Tanaka N, Suzuki S, Sakai M, Yokobori T, Nakajima M, Fukuchi M, Tsushima Y, kato H, Kuwano H. Effectiveness of FDG-PET in screening of synchronous cancer of other organs in patients with esophageal cancer. Anticancer research. 2014 Jan 1;34(1):283-7.

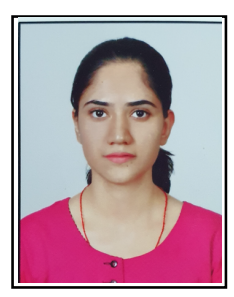

Singh K. Kanpur, Uttar Pradesh 25-02-1991. Pathology DNB IInd year trainee - Regency Hospital, Kanpur. MBBS (2016) - Era's Lucknow Medical College, Lucknow.

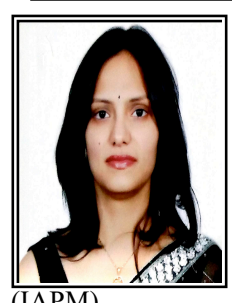

Gupta D. New Delhi 11-07-1982. MBBS, MD, DNB Pathology, DNB (2013) - NBE, MD (2011) - PGIMS Rohtak, Haryana, MBBS (2006) - LHMC, Delhi. She worked as Senior Resident at PGIMS, Rohtak and Safdarjung Hospital, Delhi before joining Regency Hospital, Kanpur, India in Nov 2012 and is working there as consultant Pathologist, since then.

Dr. Deepti Gupta is a lifetime member of

Indian Academy of Pathologists and Microbiologists (IAPM)

Indian Academy of Cytologists (IAC)

Indian Medical Association (IMA)

Dr. Deepti has one original research article and eleven case reports in indexed medical journal to her credit. 